\title{
Hybrid fluidic actuation for a foam-based soft actuator
}

\author{
Jan Peters ${ }^{1}$, Bani Anvari ${ }^{1}$, Cheng Chen $^{2}$, Zara Lim², and Helge A Wurdemann ${ }^{2}$
}

\begin{abstract}
Actuation means for soft robotic structures are manifold: despite actuation mechanisms such as tendon-driven manipulators or shape memory alloys, the majority of soft robotic actuators are fluidically actuated - either purely by positive or negative air pressure or by hydraulic actuation only. This paper presents the novel idea of employing hybrid fluidic - hydraulic and pneumatic - actuation for soft robotic systems. The concept and design of the hybrid actuation system as well as the fabrication of the soft actuator are presented: Polyvinyl Alcohol (PVA) foam is embedded inside a casted, reinforced silicone chamber. A hydraulic and pneumatic robotic syringe pump are connected to the base and top of the soft actuator. We found that a higher percentage of hydraulics resulted in a higher output force. Hydraulic actuation further is able to change displacements at a higher rate compared to pneumatic actuation. Changing between Hydraulic:Pneumatic (HP) ratios shows how stiffness properties of a soft actuator can be varied.
\end{abstract}

\section{INTRODUCTION}

In the past decades, articulated robots with rigid bodies and discrete joints have been used in industry for various manipulation, automation and motion tasks. These rigid machines are highly controllable and able to perform fast, repetitive and precise operations [1]. However, robotic systems with soft structures and actuators have been recently developed to promote safe and flexible interaction with their natural environments and with humans [2]-[4].

In some cases, soft robots with compliant bodies have been inspired by biological systems [5] which can provide robustness and adaptiveness inherently to environmental uncertainties and variations [6], [7]. These systems can deform and absorb energy arising from physical interactions and distribute applied forces. Researchers have designed and fabricated soft robots in a wide variety of shapes with infinite degrees of freedom, which can adapt to their surroundings using high-curvature bending and twisting capabilities [8], [9]. Due to their characteristics, these soft robots were applied, e.g., in the fields of abdominal minimally invasive surgery [10], [12]-[15], [30], cardiovascular interventions [16], [17] and collaborative industrial operations [18]-[20], [27].

In order to bridge the gap between soft and rigid robots, several solutions of how to change the stiffness

This work is supported by the Springboard Award of the Academy of Medical Sciences (grant number: SBF003-1109) and the Engineering and Physical Sciences Research Council (grant numbers: EP/N509577/1, EP/R037795/1, and EP/S014039/1).

${ }^{1}$ J. Peters and B. Anvari are with the Department of Civil, Environmental and Geomatic Engineering, University College London, UK. Email: b. anvari@ucl.ac.uk.

${ }^{2}$ C. Chen, Z. Lim and H.A. Wurdemann are with the Department of Mechanical Engineering, University College London, UK. Email: h. wurdemann@ucl.ac.uk.

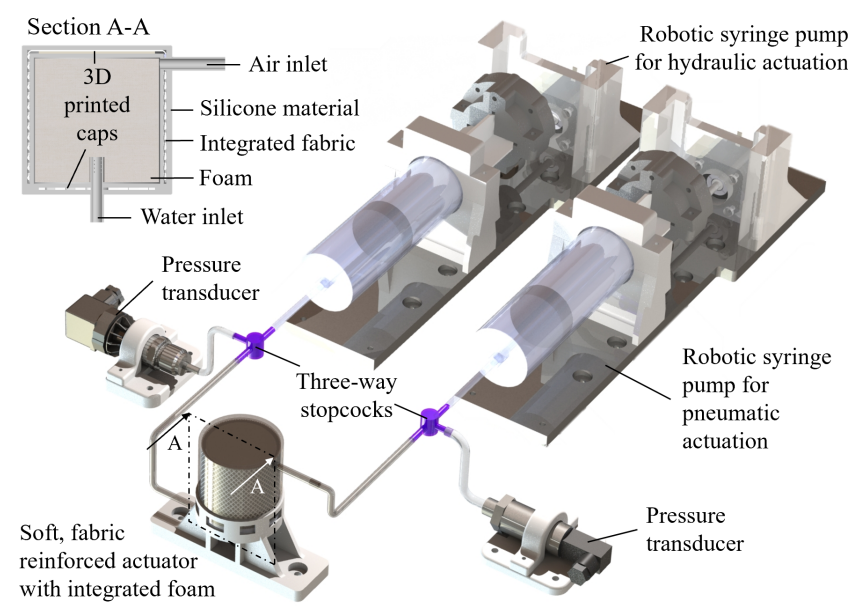

Fig. 1. Concept of the proposed hybrid fluidic actuation using a foam-based soft robotic manipulator. The soft actuator is a multi-silicone-layer cylinder filled with pre-compressed foam. Radial expansion is limited through fabric-reinforced chamber walls. The actuation system consists of two robotic syringe pumps - a pneumatic and a hydraulic one. Pressure transducers monitor the change in water and air pressure.

of soft robotic structures have been investigated in recent years [22]. Some leverage opposing actuation principles to achieve stiffness, such as [23]-[28], whilst others explore mechanisms that vary the stiffness of an entire volume of material. In [29], [30] for instance, granular jamming is integrated into an additional chamber in a silicone-based, pneumatically actuated soft manipulator. Stiffness variation can then be achieved by applying a vacuum to freeze the robots configuration in the desired position. Instead of granules, some designs utilise layer jamming methods instead, such as the concept proposed in [31] where multiple overlapping layers of thin Mylar film are used to vary stiffness in a hollow, snake-like manipulator. Application of a vacuum increases the friction between the layers to generate a change in stiffness. A Variable-Stiffness-Actuator mechanism is reported in [32] that uses the heating and cooling of a wax-coated, open-cell foam to adjust the stiffness, strength, and volume of the structures. This method introduces time delays to the changes between soft and stiff states due to the delayed response of the material to the heating and cooling processes. Alternative materials, like Low Melting Point Alloys (LMPAs), are being explored to achieve similar effects, such as in [33], [34]. Embedding LMPA into actuation chambers to stiffen the soft robot at the same time opens opportunities in miniaturisation and higher stiffness values when additional functional stiffening material is introduced into the actuation chamber [35]. 
Despite actuation mechanisms such as tendon-driven manipulators or shape memory alloys for instance [7], many of these soft robotic actuators including their stiffening mechanisms are fluidically actuated - either purely by positive [15] or negative air actuation [36] or by hydraulic actuation [37]. To the best of the authors' knowledge, there is no soft robotic actuation mechanism available that leverages the advantages of both pneumatic (i.e. its compressibility and, hence, compliance) and hydraulic (i.e. the incompressibility and its ability to exert higher forces) actuation means.

In this paper, we deliver a system that uses simultaneous hydraulic and pneumatic actuation for soft robotic manipulators as shown in Figure 1. Our system consists of two robotic syringe pumps connected to a soft, silicone-based actuator with encapsulated Polyvinyl Alcohol (PVA) foam. (Note: Our actuator has embedded thin, flexible PLA plates at the top and bottom of the manipulator to reduce ballooning effects. As the majority of materials and the overall behaviour of the robot are compliant, we consider our system to be classified as a soft robotic system.) We present experimental results comparing hydraulic with pneumatic actuation, various ratios of a combination of Hydraulic:Pneumatic (HP) actuation and when shifting from one to another means.

The overall motive for developing this hybrid fluidic actuation system for foam-based soft actuators is its specific application in transport engineering: Over the next few years, drivers will be provided with increasingly sophisticated autonomy features [38]. Each level of autonomy requires a different type/level of the drivers cognitive/physical intervention [39]. During autonomy level transitions, the vehicle may require an engaged driver to monitor the system and assume control under conditions when the car cannot drive itself [40]. We envisage arrays of foam-based, soft, stiffness-controllable robots (through changing between hydraulic and pneumatic actuation) to be integrated into driver seats transforming the seat into an ecological, intuitive haptic interface between the driver and a highly-automated vehicle. The soft robotic system based on our presented approach will be serving the feedback purpose through direct contact with the human during any level of autonomy.

Section II gives an overview of our concept. In Section III, the design, fabrication of the soft actuator and integration of the robotic syringe pumps are described. The experimental protocol, results and a discussion are presented in Section IV. Section V lists the achievements of this paper and identifies future directions of our proposed hybrid actuation principle.

\section{Proposed Methodology}

Our proposed concept as shown in Figure 1 is made of two syringe pumps supplying pneumatic air and hydraulic water pressure respectively. Pressure transducers that are embedded in each of the fluidic actuation cycles monitor the pressure. Hoses connect the syringe pumps to a soft, silicone-based manipulator. Due to the density characteristics of air and water, the hose from the pneumatic actuation syringe is connected to the manipulator's tip whereas the tube from the hydraulic pump is integrated into the base.

The hybrid actuation system is used to drive a soft, silicone-based manipulator which elongates when pressurised. The actuator is fabricated from Ecoflex 00-30 silicone and reinforced with a two-way stretchable fabric woven from $100 \%$ nylon to restrict the outward expansion of the actuator but allow elongation only. The internal chamber is filled with PVA foam, which has a high absorption and water-retention ability and is able to be used under extensive force in both wet and dry conditions.

\section{DESIGN, FABRICATION AND INTERFACING OF THE HYBRID ACTUATED SYSTEM}

\section{A. Manufacturing the silicone- and foam-based soft robot}

Figure 2 shows the design of the soft actuator, composed of several layers of silicone [Ecoflex 00-30], rectified with a two-way stretch textile which reduces the radial balloon phenomenon resulting in one-directional elongation only. Polylactic (PLA) rigid plates are used to reduce the bulge effect on the upper and lower surfaces of the actuator during operation. The internal volume of the soft robot is filled in with pre-compressed superabsorbent PVA foam. An inlet at the top provides the soft actuator with air pressure whereas the inlet at the bottom supplies water pressure.

Each of the silicone parts were produced using PLA moulds. The first layer of the main body was composed of a cylindrical $53 \mathrm{~mm}$ wide hollowed silicone chamber [Ecoflex 00-30] with an inner diameter and height of $50 \mathrm{~mm}$. The outer wall of the chamber was lined with a two-way stretch textile, allowing the actuator to extend length-ways but limiting the outward expansion of the silicone during operation. A PLA round plate [ $46 \mathrm{~mm}$ diameter, $1 \mathrm{~mm}$ thickness] was placed on top of the inner silicone chamber to reduce the ballooning effect on the upper surface. Non-stretch textile was layered

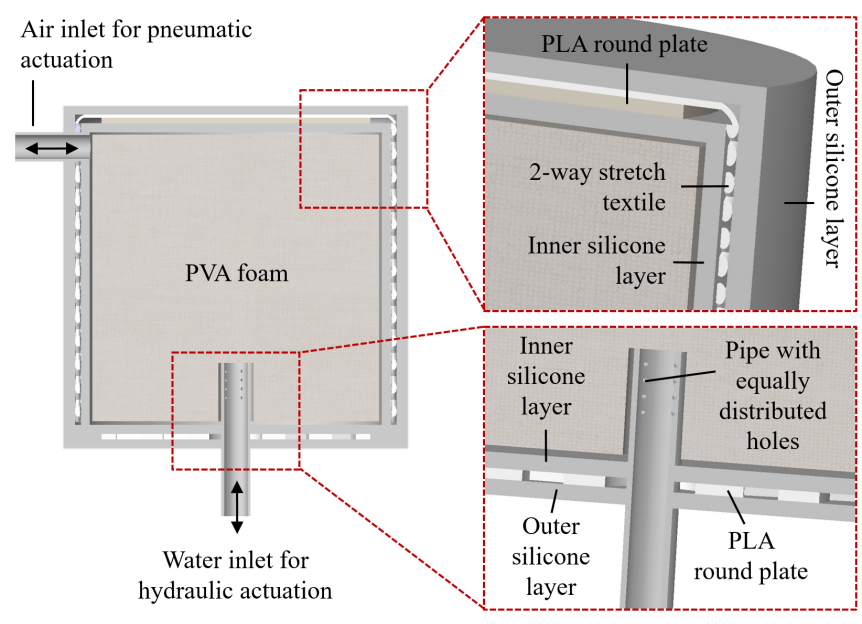

Fig. 2. The silicone-based soft actuator has a cylindrical shape with a diameter of $58 \mathrm{~mm}$ and height of $70 \mathrm{~mm}$. Two-way stretch textile is embedded between silicone layers in the wall. The top and bottom of the manipulator have enclosed Polylactic plates. The inner lumen of the actuator is filled with pre-compressed Polyvinyl Alcohol foam. Two hoses allow pneumatic and hydraulic actuation. 
on top of the rigid plate and stitched with the second layer mesh fabric to secure the second layer. A second hollow cylindrical silicone chamber was then moulded with an inner diameter of $58 \mathrm{~mm}$ and height of $64 \mathrm{~mm}$. A $3 \mathrm{~mm}$ inner diameter, $5 \mathrm{~mm}$ outer diameter silicone pipe was inserted into the wall of the second silicone layer at the upper edge secured with silicone glue for pneumatic actuation.

The base of the actuator was constructed from two layers of silicone plates [55 $\mathrm{mm}$ diameter, $6 \mathrm{~mm}$ thickness] and fortified with a rigid round plate $[45 \mathrm{~mm}$ diameter, $1 \mathrm{~mm}$ thickness] between the two silicone layers. Each of the base layer components were fabricated with a hole in the centre which was reserved for the placement of the hydraulic pipe. Pre-compressed PVA foam was then placed within the actuator, such that it can expand and evenly fill the silicone chamber as the actuator elongates. A second silicone pipe (same dimensions as the pneumatic pipe, but with additional holes drilled into the top of the pipe) was inserted through the base and $20 \mathrm{~mm}$ into the PVA foam. Finally, the silicone chamber and hydraulic silicone pipe were sealed together using silicone glue.

\section{B. Robotic interface of the hybrid actuation system}

The overall control system of the soft actuator is shown in Figure 3. The pneumatic and hydraulic pressure systems of the actuator are separately controlled using two $200 \mathrm{ml}$ syringes [SYR200 ML-LL, Romed Medical, inner barrel diameter: $46 \mathrm{~mm}$ ] which accounted for the $114 \mathrm{ml}$ volume of the soft actuator structure as well as any additional expansion that occurred as a result of using silicone. The end of each syringes are connected to the pneumatic and hydraulic silicone pipes using male luer style hose barb adapters and three-way stopcocks with luer connections. Hence, it is possible to allow either pneumatic or hydraulic actuation only by closing one three-way stopcock or hybrid actuation by opening both three-way stopcocks at the same time. The plungers of both syringes are independently driven

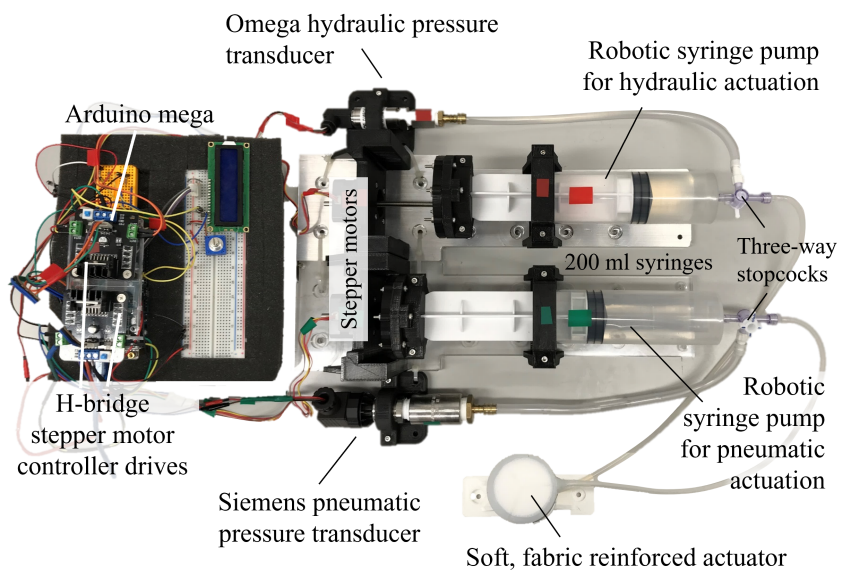

Fig. 3. The overall hybrid actuation system consists of two syringe pumps driven by stepper motors. Two transducers measure and monitor the pressure within the two fluidic cycles. Hoses connect the pneumatic and hydraulic syringe to a single compartment soft, silicone-based robotic actuator with integrated PVA foam. by stepper motors [LS4118S1404-T6X2-150, NANOTEC]. These motors incorporated a $150 \mathrm{~mm}$ screw, enabling both forwards and backwards linear motion with a driving force of up to $250 \mathrm{~N}$ which are sufficient for controlling the motion of the syringes. Each syringe plunger is affixed to the screw of the stepper motors using motor nuts [LSNUT-T6X2-F, NANOTEC] eliminating any rotary or screw movement. One full $360^{\circ}$ motor rotation, which requires 200 steps, results in a linear translation of the plunger by $2 \mathrm{~mm}$. Hence, 1 step is equivalent to a volume displacement of $0.0166 \mathrm{ml}$.

Pressure sensors monitor the effects of the hydraulic and pneumatic systems of the soft actuator. In the hydraulic system, absolute pressure is measured with the Omega PXM319, which can read values between $0-3.5$ bar with an output voltage range of $0-10 \mathrm{VDC}$. The accuracy is $0.25 \%$ when the supply voltage is $15-30$ VDC. Gauge pressure is measured using the Siemens 7MF1565-3BE00-1AA1 which can read values within a range of $0-4$ bar and produces an output current ranging from $4-20 \mathrm{~mA}$ when the power supply is between $7-33$ VDC.

Each of the main components are fixed onto a base structure constructed from several 3D printed components. Individual base and upper parts were designed to encase and secure each of the motors, pressure transducers and barrels of the syringes. Additionally, a foundation was printed for the soft actuator, allowing enough room for the pneumatic pipe to not be compressed. This ensured that the overall stability of the structure would not be affected when external forces are applied or when the actuator is being operated.

Both stepper motors are operated with a separate dual H-bridge [L298N]. An LCD displays the pressure values of each system. An Arduino Mega 2560 is used as the microcontroller to control each H-bridge via the PWM output pins, receive data from the pressure transducers via the analog pins and display the pressure values via the digital and serial communication pins. $24 \mathrm{VDC}$ is supplied to the motors and pressure transducers, and is reduced to $5 \mathrm{VDC}$ using two $250 \Omega$ resistors in order to supply the Arduino and LCD.

\section{EXPERIMENTAL EVALUATIONS}

\section{A. Experimental Procedure}

To identify the characteristics of the hybrid actuator system, three different experiments were carried out, assessing the performance of the system using various Hydraulic:Pneumatic (HP) ratios. Each of the tests can be categorised into the following three sets, where the blocked force and free-inflation displacement were measured independently.

1) Experiment 1 - 100:0 and 0:100 HP ratios: The displacement and force of the actuator were measured as it was fully controlled by either the pneumatic (with the three-way stopcock on the hydraulic circuit being closed) or the hydraulic (with the three-way stopcock on the pneumatic circuit being closed) system. The motor speed for each syringe was set at $60 \frac{\mathrm{rev}}{\mathrm{min}}$. In each case, the manipulator was initially completely deflated. For the free-inflation 
displacement test, the actuator was then inflated until the tip of the actuator reached a $12 \mathrm{~mm}$ displacement. For the blocked force test, a sensor monitored the longitudinal forces of the tip that the manipulator exerted when actuated by the same number of motor steps as during the displacement test. The actuator was then fully deflated at the same rate to the default position. The displacement and force was recorded with respect to the motor steps and pressure of each system.

2) Experiment 2 - 25:75, 50:50 and 75:25 HP ratios: The displacement and force exerted by the actuator were measured, using a fixed hybrid ratio of hydraulics and pneumatics set at 25:75, 50:50 and 75:25 HP ratios. With both three-way stopcocks being open, the pneumatics and hydraulics were simultaneously injected (by keeping the ratio of the number of steps constant) into and extracted from the actuator at set rates to maintain fixed ratios for the internal pressure of each system. The actuator was inflated recording the free-inflation displacement. For the blocked force test, the exerted forces alongside the pressure of the hydraulic and pneumatic systems was analysed as in Experiment 1.

3) Experiment 3 - 40:60 and 60:40 HP ratio swaps: With both three-way stopcocks being open, the actuator was elongated with a fixed HP ratio of either 40:60 or 60:40. Once the actuator had reached the target point, the ratios were then reversed to observe the change in stiffness and displacement. The force and displacement displacement change were recorded as described for Experiment 1. Also, the pressures of the hydraulic and pneumatic system were observed when the proportions were swapped.

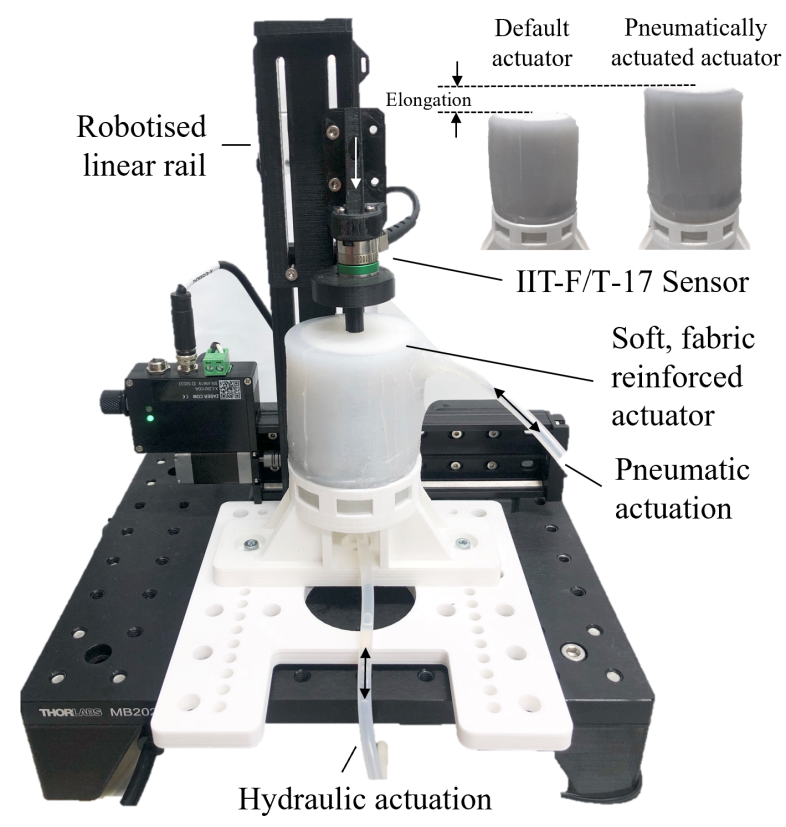

Fig. 4. The force test setup consists of the actuator being secured to a THORLAB aluminium board and a 6 DoF force/torque (F/T) sensor [IIT-FT-17] placed directly above it. As the actuator extends upwards in the z-axis, the exerted force against the sensor was recorded and collected in MATLAB.

\section{B. Experimental Setup}

For the displacement tests, the actuator was placed in an upright position with an NDI AURORA Planar Field Generator directly above. The AURORA 6 Degree-of-Freedom (DoF) position and orientation sensor was attached to the top face of the actuator and connected to a System Control Unit (SCU) via the Sensor Interface Unit (SIU). The SCU tracked the displacement of the sensor within the generated electromagnetic field, and was integrated into a MATLAB interface.

For the force tests, the base structure of the actuator was fixed to a THORLAB aluminium board (see Figure 4). A 6 DoF force/torque (F/T) sensor [IIT-FT17] was placed faced down above the actuator in its default deflated state, touching the top face without exerting any force. Force measurements were then taken to analyse the exerted forces of the manipulator during different type of actuation means for all experiments.

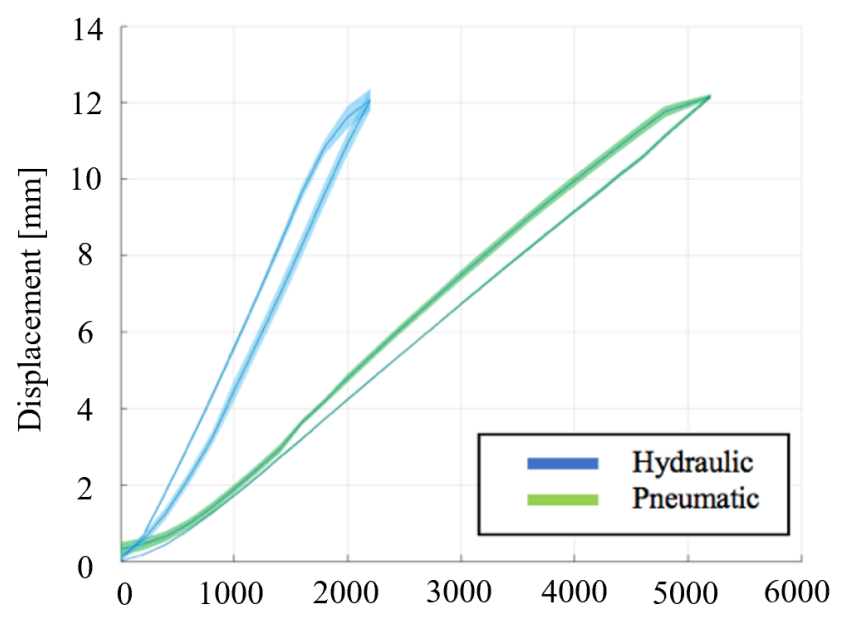

(a)

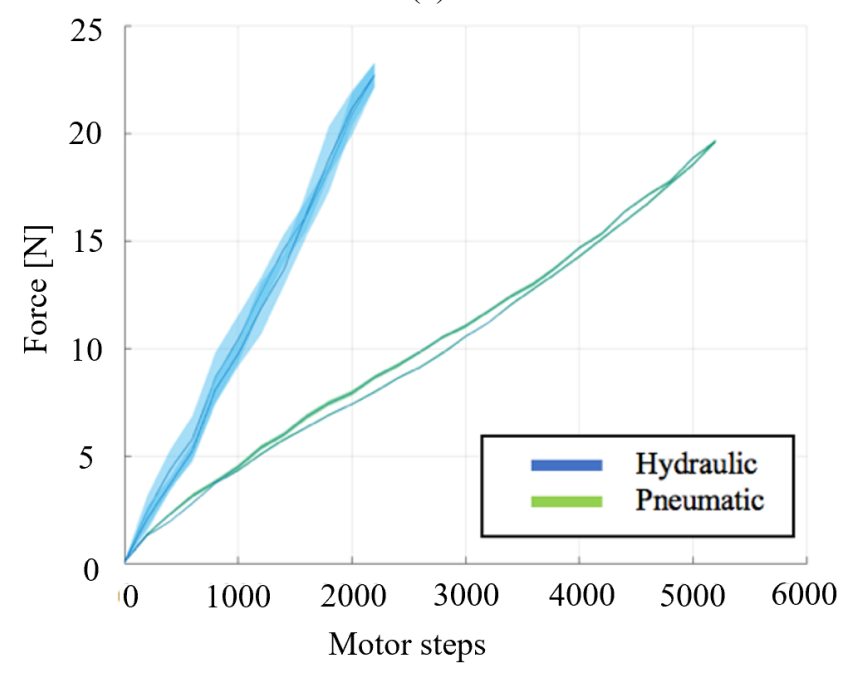

(b)

Fig. 5. Results of (a) displacement and (b) force tests controlled with 100:0 and 0:100 HP Ratios. Blue lines show the results of the actuator being fully controlled using the hydraulic system and green lines for the pneumatic system. 


\section{Experimental results}

Displacement and force measurements versus motor steps for Experiment 1 are plotted in Figure 5 showing the mean values and standard deviations of five repetitions. Blue and green lines represent the hydraulic and pneumatic actuation systems. Both tests resulted in approximately linear relationships, where the displacement tests in Figure 5 (a) showed a higher values for the hysteresis: $10 \%$ for the hydraulic system and $7.4 \%$ for the pneumatic system compared to negligible hysteresis for each of the force curves in Figure 5 (b). In both tests, the pneumatic system required a higher number of steps to achieve the same output as the hydraulic system. For instance, in order to produce a displacement of $12 \mathrm{~mm}$, the pneumatic system required 5200 steps (producing a pneumatic pressure of $1.29 \mathrm{bar}$ ), compared to the hydraulic system which only required 2200 steps, (producing a hydraulic pressure of 1.12 bar). Similarly,

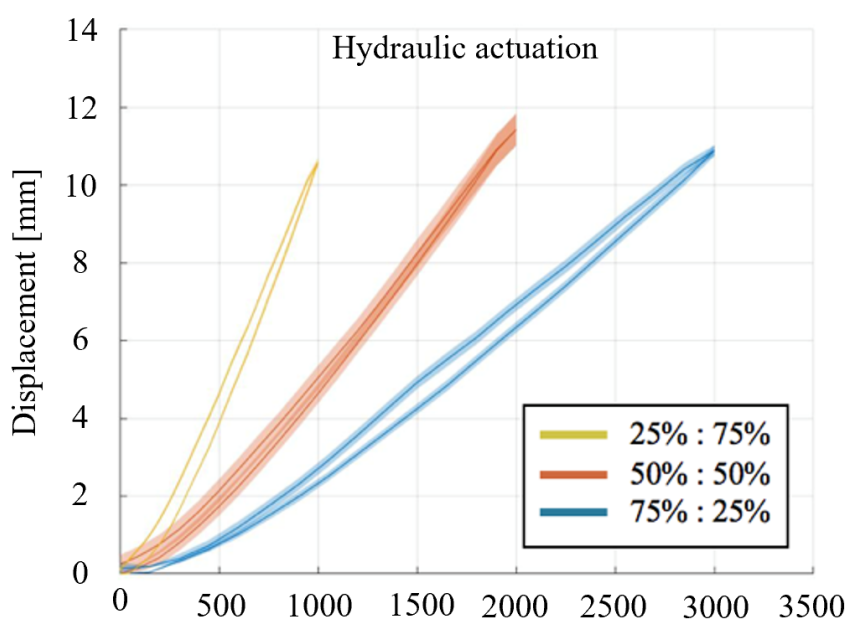

(a)

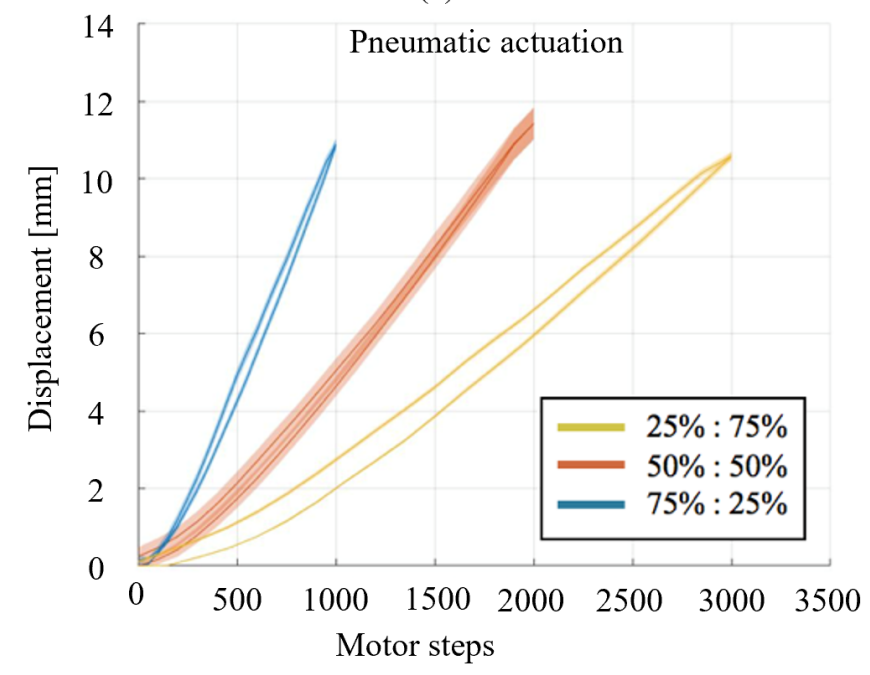

(b)

Fig. 6. Displacement tests at 25:75, 50:50 and 75:25 mixed HP ratios, where the corresponding results are represented by the yellow, red and blue lines respectively. Each graph shows the displacement of the actuator against the number of motor steps contributed by (a) the hydraulic and (b) the pneumatic actuation. for the force tests, the pneumatic system required 5000 steps (1.28bar), in order to achieve an output force of $19 \mathrm{~N}$ compared to the hydraulic system which only required 1800 steps (1.32 bar).

The results for the mixed HP ratio displacement tests are divided into two graphs - each displaying the respective number of steps that each of the systems ran to maintain the assigned HP ratios. The hydraulic results are shown in Figure 6 (a) and the pneumatic ones in Figure 6 (b). The mean and standard deviations are plotted, where the colours show the results from the displacement and the force experiments corresponding to the same ratio pair, producing the same output displacements. Yellow represents 25:75, red 50:50 and blue 75:25 HP ratio. Overall, each of the HP ratios produce approximately linear outputs with small hysteresis - for the hydraulic system, each of these values are $7.1 \%, 3.5 \%$ and $5.4 \%$ for the $25: 75,50: 50$ and

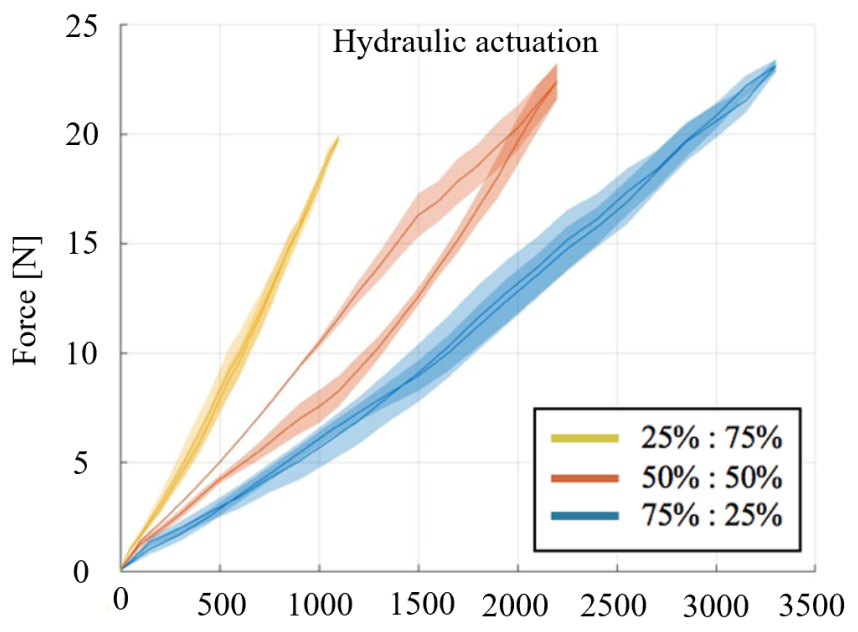

(a)

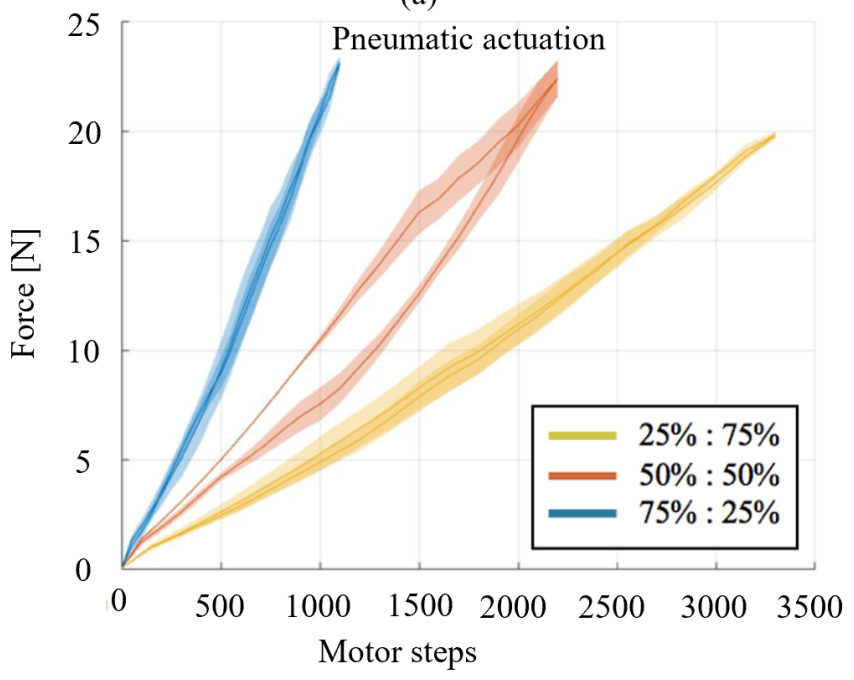

(b)

Fig. 7. Force tests at 25:75, 50:50 and 75:25 mixed HP ratios, where the corresponding results are represented by the yellow, red and blue lines respectively. Both graphs show the force of the actuator against the number of motor steps contributed by (a) the hydraulic and (b) the pneumatic actuation systems. 
75:25 HP ratios respectively; for the pneumatic system, these hysteresis values are $7.7 \%, 2.8 \%$ and $5.5 \%$. The total displacement of the actuator does not have much variance as the ratios changed. When the total number of motor steps was 4000 (corresponding to an internal pressure of 2.24bar), the displacements are $11.42 \mathrm{~mm}(50: 50 \mathrm{HP}$ ratio), $10.89 \mathrm{~mm}$ (75:25 HP ratio) and $10.59 \mathrm{~mm}$ (25:75 $\mathrm{HP}$ ratio).

The force measurements versus for the total motor steps of Experiment 2 are shown in Figure 7 (a) for hydraulic actuation and in Figure 7 (b) for pneumatic actuation. A 25:75 or 75:25 HP ratio results in a approximately linear output with a negligible hysteresis for the hydraulic and pneumatic system. The 50:50 HP ratio, however, results in a non-linear curve with a $17 \%$ hysteresis. The force values have a wider range of results for this test compared to the displacement test and increased as a higher percentage of hydraulics is used - when the total number of motor steps was 4400 (producing an internal pressure of 2.34 bar), the forces were $19.84 \mathrm{~N}$ (25:75 $\mathrm{HP}$ ratio), $22.42 \mathrm{~N}$ (50:50 HP ratio) and $23.14 \mathrm{~N}$ (75:25 $\mathrm{HP}$ ratio).

The graphs for Experiment 3 are shown in Figure 8, where the 40:60 and 60:40 HP ratio swaps are shown in Figure 8 (a), and the force graphs for the same HP ratio swaps are shown in Figure 8 (b). Here, the motor steps from each drive is represented along the $x$-axes. In both graphs, the turquoise line represents the ratio swap starting with 60:40 HP, and the purple line represents the swap starting with the 40:60 HP ratio. In Figure 8 (a), the displacement curves produce approximately linear loading outputs, matching the displacement property trends observed in Experiment 2. When the ratio was reversed when the total number of motor steps was 2600 (2.18bar), the observed displacement change was small and increased by $0.46 \mathrm{~mm}$ in both cases. The force graphs in Figure 8 (b) also produce almost linear outputs, however decrease after the ratios are reversed. When the ratios are swapped at 3000 steps $(2.23 \mathrm{bar})$, the output forces dropped by $0.48 \mathrm{~N}$ for the HP ratio starting at $60: 40$ and $0.29 \mathrm{~N}$ for the HP ratio starting at 40:60.

\section{Discussion}

The graphs from Experiment 1 in Figures 5 show that each of the pneumatic and hydraulic system produces approximately linear results when independently controlling the actuator, whereby the pneumatic system requires a larger number of steps to achieve the same result as the hydraulic system in both displacement and force. This can be accredited to the fact that air used to control the pneumatic system is more compressible than water in the hydraulic system (as air obeys the ideal gas law). This suggests that the hydraulic system is more appropriate for changing an applied force and overall displacement at a faster rate, however the pneumatic system is more suitable for achieving precision when controlling displacement or force.

Hybrid systems in Experiment 2 result in little variation in the total displacement when different HP ratios are used. This is also reflected in the hysteresis of the curves for the displacement graph as each HP ratio produce negligible values. However, force measurements for each of these $\mathrm{HP}$ ratios result in a slight increase as the percentage of hydraulics increase - this matches the results obtained from the force tests of Experiment 1. In addition, it is also observed that the HP ratios with a similar ratio of hydraulics to pneumatics resulted in a larger hysteresis, indicating that a larger difference between the two ratios results in a more consistent force output when comparing the loading and unloading cycles. This behaviour might be caused by the fact that the system was not reset between each experimental iteration.

In displacement tests of Experiment 3, the actuator's elongation slightly increases after the ratios are swapped for each case, however not by a significant amount. Similarly results are observed for force tests: the exerted force by the actuator decreases slightly in each case after the ratios are reversed - but not considerably. Both of these properties imply that when a scenario requires the actuator

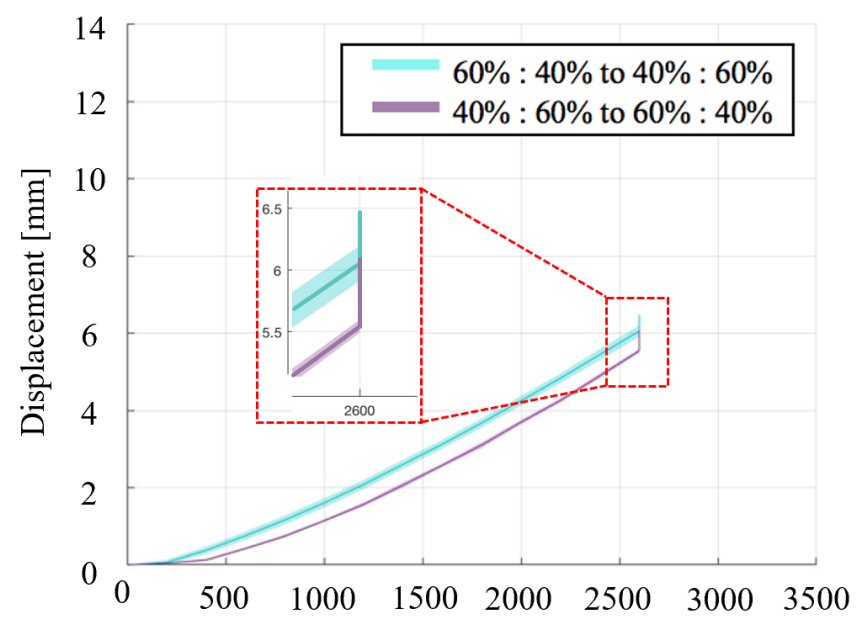

(a)

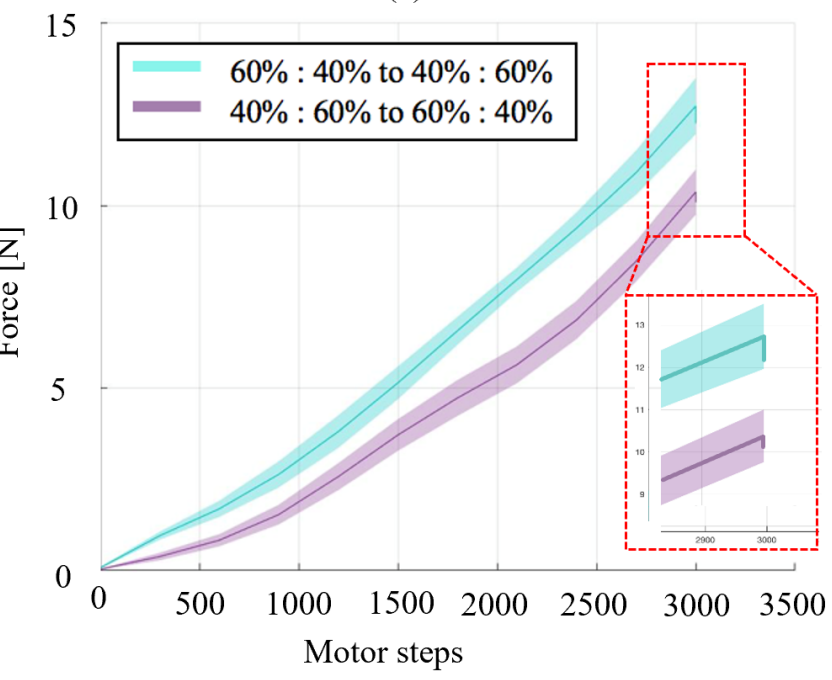

(b)

Fig. 8. (a) Displacement and (b) force graphs for the ratio swapping test starting at 40:60 and 60:40 HP ratios. The 60:40 starting HP ratio is represented by the turquoise line and the 40:60 starting ratio is represented by the purple line. 
to maintain a certain displacement but vary in stiffness, this can be achieved by simultaneously reducing the pneumatic percentage and increasing the hydraulic percentage within the actuator.

\section{CONCLUSIONS}

We have presented a novel hybrid actuation system for soft robotic actuators. Our system is able to seamlessly change the volume of hydraulics and pneumatics within the actuation chamber and, hence, vary the manipulator's stiffness and length. We discussed the design of the entire system: the construction of the soft robotic actuator, using multi-layer cast silicone chambers reinforced with two-way stretchable nylon, and the use of Polyvinyl Alcohol foam within the hydraulic chamber to evenly distribute the pressure within the actuator as well as two syringes linked to stepper motors to control the amount of hydraulics or pneumatics injected in the actuator.

Force and displacement tests were carried out on the actuator at various Hydraulic:Pneumatic (HP) ratios. Experimental results showed that a higher percentage of hydraulics resulted in a higher output force in both mixed HP ratios and also $100 \%$ hydraulic tests. In the free-inflation displacement tests, it was shown that the actuator was able to change displacements at a higher rate when fully controlled by the hydraulic system, however there was not a great variance when tested with mixed HP ratios. During the HP ratio swap tests, results only changed marginally after the ratios were reversed - for both starting HP ratios, the displacement increased and the force decreased. This suggests useful applications such as when the actuator may be required to increase in stiffness while maintaining a certain displacement and force. By using a variety of both mixed and $100 \%$ HP ratios, we can effectively change the properties of the actuator by combining the optimal characteristics from each of the hydraulic and pneumatic systems.

For future developments, we will fabricate a number of soft actuators with different embedded foam types. Studies to understand the effect on the overall displacements and force values when hydraulically and pneumatically actuated will be conducted. Stiffness variation can be modelled depending on the fabrication material combined with the ratio of hydraulic and pneumatic actuation ratio.

\section{REFERENCES}

[1] A. Grau, M. Indri, L. L. Bello and T. Sauter, "Industrial robotics in factory automation: From the early stage to the Internet of Things," Annual Conference of the IEEE Industrial Electronics Society, Beijing, pp. 6159-6164, 2017.

[2] I.D. Walker, D.M. Dawson, T. Flash, F.W. Grasso, R.T. Hanlon, B. Hochner, W.M. Kier, C.C. Pagano, C.D. Rahn, Q.M. Zhang, "Continuum robot arms inspired by cephalopods", UGVT VII, 2005.

[3] M. Cianchetti, A. Arienti, M. Follador, B. Mazzolai, P. Dario, C. Laschi, "Design concept and validation of a robotic arm inspired by the octopus", Materials Science and Engineering C, vol 31(6), pp. 1230-1239, 2011.

[4] A.D. Marchese, D. Rus, "Design, kinematics, and control of a soft spatial fluidic elastomer manipulator", International Journal of Robotics Research, vol. 35(7), pp. 840869, 2016.
[5] J. Fras, Y. Noh, H.A. Wurdemann, K. Althoefer, Soft fluidic rotary actuator with improved actuation properties, IEEE/RSJ International Conference on Intelligent Robots and Systems, pp. 5610-5615, 2017.

[6] A.D. Marchese, C.D. Onal, D. Rus, "Autonomous Soft Robotic Fish Capable of Escape Maneuvers Using Fluidic Elastomer Actuators", Soft Robotics, vol. 1(1), pp. 7587, 2014.

[7] D. Rus, M.T. Tolley, "Design, fabrication and control of soft robots, Nature, vol. 521, pp. 467-475, 2015.

[8] L.H. Blumenschein, N.S. Usevitch, B.H. Do, E.W. Hawkes, A.M. Okamura, "Helical actuation on a soft inflated robot body", IEEE International Conference on Soft Robotics, pp. 245-252, 2018.

[9] L.H. Blumenschein, L.T. Gan, J.A. Fan, A.M. Okamura, E.W. Hawkes, "A Tip-Extending Soft Robot Enables Reconfigurable and Deployable Antennas", IEEE Robotics and Automation Letters, vol. 3(2), pp. 949-956, 2018.

[10] M. Cianchetti, T. Ranzani, G. Gerboni, I. de Falco, C. Laschi, A. Menciassi, "STIFF-FLOP Surgical Manipulator: Mechanical Design and Experimental Charaterization of the Single Module", IEEE/RSJ International Conference on Intelligent Robots and Systems, pp. 3576-3581, 2013.

[11] M. Li, H. Liu, A. Jiang, L.D. Seneviratne, P. Dasgupta, K. Althoefer, H.A. Wurdemann, "Intra-operative tumour localisation in robot-assisted minimally invasive surgery: A review", Journal of Engineering in Medicine, vol. 228(5), pp. 509-522, 2014.

[12] J. Fras, J. Czarnowski, M. Macias, J. Glowka, M. Cianchetti, A. Menciassi, "New STIFF-FLOP module construction idea for improved actuation and sensing", IEEE International Conference on Robotics and Automation, pp. 2901-2906, 2015.

[13] A. Arezzo, Y. Mintz, M.E. Allaix, S. Arolfo, M. Bonino, G. Gerboni, M. Brancadoro, M. Cianchetti, A. Menciassi, H.A. Wurdemann, Y. Noh, K. Althoefer, J. Fras, J. Glowka, Z. Nawrat, G. Cassidy, R. Walker, M. Morino, "Total mesorectal excision using a soft and flexible robotic arm: a feasibility study in cadaver models, Surgical Endoscopy, vol. 31(1), pp. 264273, 2016.

[14] A. Althoefer, H.A. Wurdemann, A. Shafti, A. Shiva, J. Konstantinova, "Soft and Stiffness-controllable Robotics Solutions for Minimally Invasive Surgery The STIFF-FLOP Approach", River Publishers Series in Automation, Control and Robotics, April 2018.

[15] A. Shiva, H. Sadati, Y. Noh, J. Fras, A. Ataka, H. Wurdemann, H. Hauser, I. Walker, T. Nanayakkara, K. Althoefer, "Elasticity vs. Hyperelasticity Considerations in Quasi-Static Modelling of a Soft Finger-like Robotic Appendage for Realtime Position \& Force Estimation", Soft Robotics, vol. 6 (2), 2019.

[16] E.H. Murai, S. Homer-Vanniasinkam, P.G. Silveira, J.S. Dai, D. Martins, H.A. Wurdemann, "Towards a modular suturing catheter for minimally invasive vascular surgery", IEEE International Conference on Robotics and Automation, pp. 44-49, 2018.

[17] A. Palombi, G.M. Bosi, S. Di Giuseppe, E. De Momi, S Homer-Vanniasinkam, G. Burriesci, H.A. Wurdemann, "Sizing the aortic annulus with a robotised, commercially available soft balloon catheter: in vitro study on idealised phantoms", in IEEE International Conference on Robotics and Automation, 2019.

[18] A. Stilli, H.A. Wurdemann, K. Althoefer, "A Novel Concept for Safe, Stiffness-Controllable Robot Links", Soft Robotics, vol. 4(1), pp. 16-22, 2016.

[19] A. Stilli, L. Grattarola, H. Feldmann, H.A. Wurdemann, K. Althoefer, "Variable Stiffness Links VSL Toward Inherently Safe Robotic Manipulators", in IEEE International Conference on Robotics and Automation, pp. 4971-4976, 2017.

[20] J.M. Gandarias, Y. Wang, A. Stilli, A.J. Garcia-Cerezo, J.M Gomez-de-Gabriel, H.A. Wurdemann, "Open-loop position control in collaborative, modular Variable-Stiffness-Link (VSL) robots", IEEE Robotics and Automation Letters, vol. 5(2), pp. 1772-1779, 2020.

[21] A. Stilli, A. Cremoni, M. Bianchi, A. Ridolfi, F. Gerli, F. Vannetti, H.A. Wurdemann, B. Allotta, K. Althoefer, "AirExGlove A Novel Pneumatic Exoskeleton Glove for Adaptive Hand Rehabilitation in Post-Stroke Patients", IEEE International Conference on Soft Robotics, pp. 579-584, 2018.

[22] M. Manti, V. Cacucciolo, M. Cianchetti, "Stiffening in Soft Robotics: A Review of the State of the Art", IEEE Robotics and Automation Magazine, vol. 23(3), pp. 93-106, 2016.

[23] A. Stilli, H.A. Wurdemann, K. Althoefer, "Shrinkable, stiffness-controllable soft manipulator based on a bio-inspired antagonistic actuation principle", IEEE/RSJ International Conference 
on Intelligent Robots and Systems, Chicago, USA, pp. 2476-2481, 2014.

[24] F. Maghooa, A. Stilli, K. Althoefer, H.A. Wurdemann, "Tendon and pressure actuation for a bio-inspired manipulator based on an antagonistic principle", IEEE International Conference on Robotics and Automation, pp. 2556-2561, 2015.

[25] H.A. Wurdemann, A. Stilli, K. Althoefer, "An antagonistic actuation technique for simultaneous stiffness and position control", in Lecture Notes in Computer Science: An Antagonistic Actuation Technique for Simultaneous Stiffness and Position Control, Springer, pp. 164-174, 2015.

[26] A. Shiva, A. Stilli, Y. Noh, A. Faragasso, K. Althoefer and H.A. Wurdemann, "Tendon-based stiffening for a pneumatically actuated soft manipulator", IEEE Robotics and Automation Letters, vol. 1(2), pp. 632-637, 2016.

[27] A. Stilli, K. Althoefer, H.A. Wurdemann, "Soft Robotics. Bio-inspired Antagonistic Stiffening", Developing Support Technologies, Biosystems \& Biorobotics, Springer, pp. 207-214, 2018.

[28] A. Gallarello, A. Palombi, G. Annio, S. Homer-Vanniasinkam, E. De Momi, G. Maritati, R. Torii, G. Burriesci, H.A. Wurdemann, "Patient-specific aortic phantom with tuneable compliance", ASME Journal of Engineering and Science in Medical Diagnostics and Therapy, vol. 2(4), pp. 041005, 2019.

[29] A. Jiang, G. Xynogalas, P. Dasgupta, K. Althoefer, T. Nanayakkara, "Design of a variable stiffness flexible manipulator with composite granular jamming and membrane coupling", IEEE/RSJ International Conference on Intelligent Robots and Systems, pp. 2922-2927, 2012.

[30] M. Li, T. Ranzani, S. Sareh, L.D. Seneviratne, P. Dasgupta, H.A. Wurdemann, K. Althoefer, "Multi-fingered haptic palpation utilising granular jamming stiffness feedback actuators", Smart Materials And Structures, vol. 23(9), pp. 1-11, 2014.

[31] Y.J. Kim, S. Cheng, S. Kim, K. Iagnemma, "Design of a Tubular Snake-like Manipulator with Stiffening Capability by Layer Jamming", IEEE/RSJ International Conference on Intelligent Robots and Systems, pp. 4251-4256, 2012.

[32] N.G. Cheng, A. Gopinath, L. Wang, K. Iagnemma, A.E. Hosoi, "Thermally Tunable, Self-Healing Composites for Soft Robotic Applications", Macromolecular Materials and Engineering, vol. 299(11), pp. 1279-1284, 2014.

[33] J. Shintake, B. Schubert, S. Rosset, H. Shea, D. Floreano, "Variable stiffness actuator for soft robotics using dielectric elastomer and low-melting-point alloy," IEEE/RSJ International Conference on Intelligent Robots and Systems, pp. 1097-1102, 2015.

[34] H. Abidi, A. Tonazzini, M. Cianchetti, D. Floreano, A. Menciassi, "Low Melting Point Alloy Based Stiffening of a Soft Robot", International Congress of the Society for Medical Innovation and Technology, 2017.

[35] J. Peters, E. Nolan, M. Wiese, M. Miodownik, S. Spurgeon, A. Arezzo, A. Raatz, H.A. Wurdemann, "Actuation and stiffening in fluid-driven soft robots using low-melting-point material", in IEEE/RSJ International Conference on Intelligent Robots and Systems, 2019.

[36] M.A. Robertson, J. Paik, "Low-inertia vacuum-powered soft pneumatic actuator coil characterization and design methodology", in IEEE International Conference on Soft Robotics, pp. 431-436, 2018.

[37] L. Lindenroth, J. Back, A. Schoisengeier, Y. Noh, H.A. Wurdemann, K. Althoefer, H. Liu, "Stiffness-Based Modelling of a Hydraulically-Actuated Soft Robotics Manipulator", in IEEE/RSJ International Conference on Intelligent Robots and Systems, pp. 2458-2463, 2016.

[38] I. Yaqoob, K. Hee, L.U. Khan, S.M. Ahsan Kazmi, M. Imran, N. Guizani, C.S. Hong "Autonomous Driving Cars in Smart Cities: Recent Advances, Requirements, and Challenges", IEEE Network, vol. 34(1), pp. 174-181, 2020.

[39] J.-L. Kaestle, B. Anvari, J. Krol, H.A. Wurdemann, "Correlation between Situational Awareness and EEG signals", 2020.

[40] N.A. Stanton, P.M. Salmon, G.H. Walker, E. Salas, P.A. Hancock, "State-of-science: Situation awareness in individuals, teams and systems", Ergonomics, vol. 60(4), pp. 449-466, 2017. 